\title{
A composite functional equation from algebraic aspect
}

\author{
PÁl Burai, Attila Házy and Tibor Juhász
}

Abstract. In this paper we discuss the composite functional equation

$$
f(x+2 f(y))=f(x)+y+f(y)
$$

on an Abelian group. This equation originates from Problem 10854 of the American Mathematical Monthly. We give an algebraic description of the solutions on uniquely 3-divisible Abelian groups, and then we construct all solutions $f$ of this equation on finite Abelian groups without elements of order 3 and on divisible Abelian groups without elements of order 3 including the additive group of real numbers.

Mathematics Subject Classification (2010). 39B12, 39B52.

Keywords. Composite functional equation, iterative functional equation, additive mapping, automorphism, Hamel basis, Abelian group.

\section{Introduction}

Solving composite functional equations is a popular topic nowadays (see e.g. $[1-3,5,9,12-14,17])$ in spite of the fact that they are harder to solve than a non-composite one in general. In most cases some additional assumptions on the unknown function are required to determine solutions or some "improving regularity" type theorems are needed (se e.g. $[7,11,15,16]$ ).

Our main goal is to solve a composite type functional equation on Abelian groups. The original problem was posed in 2001 in the American Mathematical Monthly [4].

10854. Proposed by Wu Wei Chao, Guang Zhou Normal University, Guang Zhou City, China. Find every function $f: \mathbb{R} \rightarrow \mathbb{R}$ that is continuous at 0 and satisfies

This research has been supported by the Hungarian Scientific Research Fund (OTKA) Grant NK-81402 and OTKA "Mobility" call Human-MB08A-84581. The second-named author's research was supported by the János Bolyai Research Scholarship of the Hungarian Academy of Sciences. 


$$
f(x+2 f(y))=f(x)+y+f(y)
$$

for all real numbers $x$ and $y$.

The solution was published in 2004 by Henderson [8]. He showed first that every solution is additive, and so $f(x)=c x$, where $c=1$ or $c=-\frac{1}{2}$.

In 2006, Boros and Daróczy [2] generalized the problem, and they described the nowhere continuous solutions of (1). Actually, they considered $\mathbb{R}$ as a vector space over $\mathbb{Q}$, and proved that $f$ is a solution of (1) if and only if $f$ is additive, and there exist a Hamel basis $H$ of $\mathbb{R}$ and a mapping $\varrho: H \rightarrow\left\{-\frac{1}{2}, 1\right\}$ such that $f(h)=\varrho(h) h$ for every $h \in H$. In this paper we investigate Eq. (1) for the case when the unknown function $f$ is defined on an arbitrary Abelian group.

Problem 1. Let $G$ be an Abelian group under addition. Find every function $f: G \rightarrow G$ satisfying

$$
f(x+2 f(y))=f(x)+y+f(y)
$$

for all $x, y \in G$.

We will show that every solution of (2) is an automorphism of the group $G$, and describe the solutions for the case when $G$ is uniquely 3-divisible; that is, when for any $a \in G$ there is exactly one $x \in G$ with $3 x=a$. Since the additive group of $\mathbb{R}$, or more generally, the underlying group of any vector space over $\mathbb{Q}$ is uniquely 3-divisible, the results mentioned above of Henderson, and Boros and Daróczy are rediscovered. Furthermore, a finite Abelian group with no elements of order 3 is also uniquely 3 -divisible, and it has a basis (in group theoretical sense), so we may expect an analogous result to that of Boros and Daróczy for this case. At this stage we should clarify what we mean by a basis of a group. The nonzero elements $x_{1}, \ldots, x_{k}$ of an Abelian group $G$ are said to be independent, if for any integers $n_{1}, \ldots, n_{k}$ the equality $n_{1} x_{1}+\cdots+n_{k} x_{k}=0$ implies $n_{1} x_{1}=\cdots=n_{k} x_{k}=0$; that is, $n_{i}=0$ if the order $o\left(x_{i}\right)$ of $x_{i}$ is infinite, and $n_{i} \equiv 0\left(\bmod o\left(x_{i}\right)\right)$ if $o\left(x_{i}\right)$ is finite. A set of elements $H=\left\{x_{\alpha}\right\}_{\alpha \in \Gamma}$ of $G$, where $\Gamma$ is an index set of arbitrary power, is called independent, if any finite subset of $H$ is independent. Evidently, a subset $H=\left\{x_{\alpha}\right\}_{\alpha \in \Gamma}$ of $G$ is independent if and only if the subgroup generated by $H$ is the direct sum of the cyclic groups $\left\langle x_{\alpha}\right\rangle$. If $H$ is an independent generating system of $G$, then we say that $H$ is a basis of $G$. At first glance, there may be an analogy between this concept and the basis in a vector space, but there are some significant differences. For example, the set $\{2\}$ is a maximal independent set (there is no independent set containing it properly) of $\mathbb{Z}$, but it is not a basis of $\mathbb{Z}$, so a maximal independent set is not always a basis. This is why the method of the proof used by Boros and Daróczy does not work for finite Abelian groups, and we need another approach. 


\section{Results}

Throughout this paper $G$ means an Abelian group written additively. For a subset $H$ of $G,\langle H\rangle$ denotes the subgroup of $G$ generated by $H$.

Theorem 1. The function $f: G \rightarrow G$ is a solution of the functional equation (2) if and only if $f$ is an automorphism of the group $G$ such that

$$
2 f(f(y))=y+f(y)
$$

holds for all $y \in G$.

Proof. We claim first that $f(0)=0$. Substituting $x=y=0$ into (2) we have that

$$
f(2 f(0))=2 f(0) .
$$

Furthermore, under $x=2 f(0)$ and $y=0$, (2) yields $f(4 f(0))=3 f(0)$, and setting $y=2 f(0)$ and $x=0$, we have that $f(4 f(0))=5 f(0)$. These together show that $3 f(0)=5 f(0)$; that is, $2 f(0)=0$ and the claim follows from (4).

We show now that $f$ is an odd function. For $x=0$ from (2) we get that $f(2 f(y))=y+f(y)$ for all $y \in G$. Hence

$$
\begin{aligned}
f(2 f(x)+2 f(y)) & =f(2 f(x))+y+f(y) \\
& =x+f(x)+y+f(y) .
\end{aligned}
$$

Let $b=f(x)+f(-x)$ for some fixed $x \in G$. From the equation above it follows that $f(2 b)=b$, whence $f(2 f(2 b))=f(2 b)$. But as we have already seen, $f(2 f(2 b))=2 b+f(2 b)$. Thus, $2 b=0$. Using that $f(2 b)=b$, we get that $b=0$, which means that $f$ is an odd function.

For fixed $x, y \in G$ let

$$
c(x, y)=f(x+y)-f(x)-f(y) .
$$

Then by (2),

$$
\begin{aligned}
f(2 c(x, y)) & =f(-2(f(x)+f(y))+2 f(x+y)) \\
& =f(-2(f(x)+f(y)))+x+y+f(x+y) .
\end{aligned}
$$

Using the oddness of the function $f$ and (5),

$$
\begin{aligned}
f(-2(f(x)+f(y))) & =-f(2 f(x)+2 f(y)) \\
& =-x-f(x)-y-f(y),
\end{aligned}
$$

and so $f(2 c(x, y))=c(x, y)$. Similarly as before,

$$
f(2 f(2 c(x, y)))=f(2 c(x, y)),
$$

and with $x=0$ in (2),

$$
f(2 f(2 c(x, y)))=2 c(x, y)+f(2 c(x, y)) .
$$


Hence $2 c(x, y)=0$. In view of $f(2 c(x, y))=c(x, y)$, we proved that $c(x, y)=0$; that is, $f$ is an endomorphism.

Applying the additivity of $f$ for (2), we have that

$$
2 f(f(y))=y+f(y)
$$

for all $y \in G$. Hence, assuming that $f(y)=0$, it follows that $y=0$, thus $f$ is injective.

In what follows, we will use freely the easy fact that a group automorphism preserves the order of the group elements.

Theorem 2. All elements of 2-power order of $G$ are invariant under functions $f: G \rightarrow G$ satisfying (2).

Proof. If $y \in G$ has order 2, then the left-hand side of (3) is zero, thus $0=$ $y+f(y)$, that is $f(y)=y$. Assume by induction for some $n>1$ that each element $x \in G$ of order $2^{n-1}$ satisfies the equality $f(x)=x$. Let $y \in G$ be of order $2^{n}$. Then $o(2 f(f(y)))=2^{n-1}$ and by (3), o $o(y+f(y))=2^{n-1}$. The inductive hypothesis and the additivity of $f$ force

$$
y+f(y)=f(y+f(y))=f(y)+f(f(y)),
$$

whence $y=f(f(y))$ follows. If we substitute this into (3) the proof will be complete.

Let $a$ be an element of $G$ and $n$ be an integer. We say that $a$ is divisible by $n$, if there exists $x \in G$ such that $n x=a$. For example, if $a$ is of finite order, and $n$ is relatively prime to $o(a)$, then there exist integers $s$ and $t$ such that $1=n s+o(a) t$. The element $x=s a$ satisfies $n x=n s a=a-o(a) t a=a$, therefore $a$ is divisible by $n$. In general, the solution $x$ of the equation $n x=a$ may not be unique, the set of all solutions is the coset $x+G[n]$, where $G[n]$ denotes the set of all $g \in G$ with $n g=0$. Let $p$ be a prime. We shall say that $G$ is $p$-divisible, if each of its elements is divisible by $p$. Furthermore, if $G$ is $p$-divisible, and $G[p]=\{0\}$, then $G$ will be called uniquely $p$-divisible, and for $a \in G$ and integer $m$, the only one solution of the equation $p x=m a$ will be denoted by the symbol $\frac{m}{p} a$. In this way, a uniquely $p$-divisible group can be considered as a module over the ring $R_{p}$ of rational numbers with denominator a power of $p$. Finally, $G$ is said to be (uniquely) divisible, if it is (uniquely) divisible for every prime $p$.

For the integer $m$ and automorphism $f$ of the group $G$ let

$$
G_{m}^{f}=\{x \in G: m f(x)=x\} .
$$

Evidently, $G_{m}^{f}$ is a subgroup of $G$. Furthermore, if $G$ is uniquely $p$-divisible, then so is $G_{m}^{f}$. Indeed, for any $b \in G_{m}^{f}$ there exists exactly one $x_{0} \in G$, such that $p x_{0}=b$. Then

$$
p\left(m f\left(x_{0}\right)\right)=m f\left(p x_{0}\right)=m f(b)=b .
$$

By the uniqueness of $x_{0}$ we have $m f\left(x_{0}\right)=x_{0}$, which proves that $x_{0} \in G_{m}^{f}$. 
Theorem 3. Let $G$ be a uniquely 3-divisible group. Then the automorphism $f: G \rightarrow G$ satisfies $E q$. (3) for every $x \in G$ if and only if $G$ is a direct sum of $G_{1}^{f}$ and $G_{-2}^{f}$.

Proof. Assume first that $f$ fulfils (3). Let $x_{0}$ be an arbitrary element of $G$, and let $u=x_{0}+2 f\left(x_{0}\right)$ and $v=x_{0}-f\left(x_{0}\right)$. Then by $(3)$,

$$
f(u)=f\left(x_{0}+2 f\left(x_{0}\right)\right)=f\left(x_{0}\right)+2 f\left(f\left(x_{0}\right)\right)=x_{0}+2 f\left(x_{0}\right)=u,
$$

and

$$
-2 f(v)=-2 f\left(x_{0}-f\left(x_{0}\right)\right)=-2 f\left(x_{0}\right)+2 f\left(f\left(x_{0}\right)\right)=x_{0}-f\left(x_{0}\right)=v,
$$

thus $u \in G_{1}^{f}$ and $v \in G_{-2}^{f}$. Moreover, $G_{1}^{f}$ and $G_{-2}^{f}$ are uniquely 3-divisible, so there exist $\frac{1}{3} u \in G_{1}^{f}$ and $\frac{2}{3} v \in G_{-2}^{f}$. Using that $G$ is an $R_{p}$-module, we have that $x_{0}=\frac{1}{3} u+\frac{2}{3} v$, and so $G=\left\langle G_{1}^{f}, G_{-2}^{f}\right\rangle$. Furthermore, if $x \in G_{1}^{f} \cap G_{-2}^{f}$, then $3 f(x)=0$. Since $G$ is uniquely 3-divisible and $f$ is injective, we get that $x=0$. So, $G=G_{1}^{f} \oplus G_{-2}^{f}$, as desired.

Conversely, if $G=G_{1}^{f} \oplus G_{-2}^{f}$, then every $x \in G$ can be (uniquely) written as $x=a+b$, where $a \in G_{1}^{f}$ and $b \in G_{-2}^{f}$. Since $f$ is an automorphism, we have that

$$
2 f(f(x))=2 f(f(a))+2 f(f(b))=2 a-f(-2 f(b))=2 a-f(b)
$$

and

$$
x+f(x)=a+b+f(a)+f(b)=a-2 f(b)+a+f(b)=2 a-f(b) .
$$

Therefore, $2 f(f(x))=x+f(x)$ for every $x \in G$, and $f$ is a solution of (3).

Example 1. Let

$$
G=\langle a \mid 3 a=0\rangle \oplus\langle b \mid 3 b=0\rangle \cong \mathbb{Z}_{3} \oplus \mathbb{Z}_{3} .
$$

Then the automorphism $f$ of $G$ sending $a$ to $a+b$ and $b$ to $b$ fulfils (2), and $G_{1}^{f}=G_{-2}^{f}=\langle b\rangle$. Therefore, Theorem 3 is no longer true for groups containing elements of order 3 .

Now, we can present various possibilities by describing all solutions of the functional equation (2) for some classes of Abelian groups.

\section{1. $G$ is a finite Abelian group with no elements of order 3}

In this case the order of every element of $G$ is relatively prime to 3 , therefore $G$ is uniquely 3 -divisible. Let $f$ be a solution of (2) and $a \in G_{-2}^{f}$. Then, by Theorem 2, $a$ is of odd order. Furthermore, both $f(a)$ and $a_{0}=\frac{o(a)-1}{2} a$ are solutions of the equation $-2 x=a$, thus $f(a)=a_{0}+u$ for some $u \in G[2]$. Since $f$ is an automorphism, and $o\left(a_{0}\right)=o(a)$, we have 


$$
o(a)=o(f(a))=o\left(a_{0}+u\right)=o\left(a_{0}\right) o(u)=o(a) o(u),
$$

which implies that $u=0$; that is $f(a)=\frac{o(a)-1}{2} a$.

According to the fundamental theorem of finite Abelian groups, both $G_{1}^{f}$ and $G_{-2}^{f}$ can be expressed as a direct sum of a finite number of cyclic groups of prime power order, so we can state the following description.

Corollary 1. Let $G$ be a finite Abelian group with no elements of order 3. The function $f: G \rightarrow G$ fulfils Eq. (2) for every $x \in G$ if and only if $f$ is an automorphism of $G$, and there exist a basis $\left\{h_{1}, \ldots, h_{r}\right\}$ of $G$ and a subset $\Gamma \subset\{1, \ldots, r\}$ such that $f\left(h_{i}\right)=h_{i}$ for every $i \in \Gamma$, and $f\left(h_{i}\right)=\frac{o\left(h_{i}\right)-1}{2} h_{i}$ for $i \in \Gamma \backslash\{1, \ldots, r\}$, provided that if $o\left(h_{i}\right)$ is even, then $i \in \Gamma$.

As Example 1 shows, Corollary 1 is not true for finite Abelian groups with elements of order 3 .

\section{2. $G=\mathbb{Z}\left(p^{\infty}\right)$, the Prüfer $p$-group with $p \neq 3$}

The Prüfer $p$-group is the $p$-primary component of the factor group $\mathbb{Q} / \mathbb{Z}$, and it may be generated by $x_{1}, x_{2}, x_{3}, \ldots, x_{n}, \ldots$ so that $x_{1} \neq 0$ and

$$
p x_{1}=0, p x_{2}=x_{1}, p x_{3}=x_{2}, \ldots, p x_{n+1}=x_{n}, \ldots
$$

This group $\mathbb{Z}\left(p^{\infty}\right)$ is divisible, moreover it is uniquely $q$-divisible by every prime $q \neq p$, and each of its proper subgroup is cyclic. However, $\mathbb{Z}\left(p^{\infty}\right)$ cannot be the direct sum of two cyclic groups, so for $p>3$ we get that either $G=G_{1}^{f}$ or $G=G_{-2}^{f}$, which shows that Eq. (2) has two solutions:

$$
f(x)=x \quad \text { and } \quad f(x)=-\frac{1}{2} x \quad\left(x \in \mathbb{Z}\left(p^{\infty}\right)\right) .
$$

In the case when $p=2$, the only solution is the identity map.

\section{3. $G$ is a uniquely divisible group}

It is easy to see that any uniquely divisible group admits the canonical structure of a vector space over $\mathbb{Q}$, so Hamel's statement that any vector space has a basis can be interpreted as $G$ being a direct sum of groups each isomorphic to the additive group of rational numbers. In this way, Theorem 2 of [2] follows from Theorem 3.

Corollary 2. Let $G$ be a uniquely divisible group. The function $f: G \rightarrow G$ fulfils $E q$. (2) for every $x \in G$ if and only if $f$ is additive, and there exist $a \mathbb{Q}$-basis $H$ of $G$ and a mapping $\varrho: H \rightarrow\left\{-\frac{1}{2}, 1\right\}$ such that $f(h)=\varrho(h) h$ for every $h \in H$. 
2.3.1. $G$ is the additive group of $\mathbb{Q} \cdot \mathbb{Q}$ is uniquely divisible, and it cannot be expressed as the internal direct sum of two proper subgroups. So, either $G=G_{1}^{f}$ or $G=G_{-2}^{f}$, and the only two solutions of Eq. (2) are:

$$
f(x)=x \quad \text { and } \quad f(x)=-\frac{1}{2} x \quad(x \in \mathbb{Q}) .
$$

2.3.2. $G$ is the additive group of $\mathbb{R}$. $\mathbb{R}$ is also a uniquely divisible group, so Eq. (2) has infinitely many solutions, and by Corollary 5.2.1. of [10], exactly two of them are continuous at 0 , namely when the mapping $\varrho$ is constant. Thus, the solution for the original problem is obtained.

In view of the structure theorem on divisible groups (see Theorem 19.1. of [6]), a divisible group is a direct sum of Prüfer groups and the additive group of rationals, therefore Cases 2.2 and 2.3 together give all the solutions of (2) for divisible groups with no elements of order 3 .

However, for groups containing elements of order 3 the problem remains open.

\section{References}

[1] Aczél, J., Dhombres, J.: Functional equations in several variables. Cambridge University Press, Cambridge (1989)

[2] Boros, Z., Daróczy, Z.: A composite functional equation with additive solutions. Publ. Math. Debrecen 69(1-2), 245-253 (2006)

[3] Daróczy, Z., Páles, Zs.: Gauss-composition of means and the solution of the MatkowskiSutô problem. Publ. Math. Debrecen 61(1-2), 157-218 (2002)

[4] Chao, W.W.: Problem 10854. Amer. Math. Monthly 108(2), 171 (2001)

[5] Fechner, W.: On a composite functional equation on Abelian groups. Aequationes Math. 78(1-2), 185-193 (2009)

[6] Fuchs, L.: Abelian groups. Publishing House of the Hungarian Acedemy of Sciences, Budapest (1958)

[7] Gilányi, A., Páles, Zs.: A regularity theorem for composite functional equations. Arch. Math. (Basel) 77, 317-322 (2001)

[8] Henderson, D.: Continuous additive functions; solution to Problem 10854. Amer. Math. Monthly 111(8), 725-726 (2004)

[9] Kochanek, T.: On a composite functional equation fulfilled by modulus of an additive function. Aequationes Math. 80(1-2), 155-172 (2010)

[10] Kuczma, M.: In: Gilányi, A. (ed.) An introduction to the theory of functional equations and inequalities, 2nd edn. Birkhäuser, Basel (2009)

[11] Járai, A.: Regularity properties of functional equations in several variables. Advances in Mathematics, vol. 8. Springer, New York (2005)

[12] Maksa, Gy., Páles, Zs.: On a composite functional equation arising in utility theory. Publ. Math. Debrecen 65(1-2), 215-221 (2004)

[13] Matkowski, J., Okrzesik, J.: On a composite functional equation. Demonstratio Math. 36(3), 653-658 (2003)

[14] Okrzesik, J.: On the existence of Lipschitzian solution of a composite functional equation. Demonstratio Math. 39(1), 97-100 (2006)

[15] Páles, Zs.: A regularity theorem for composite functional equations. Acta Sci. Math. (Szeged) 69(3-4), 591-604 (2003) 
[16] Páles, Zs.: Regularity problems and results concerning composite functional equations. Tatra Mt. Math. Publ. 34, part II, 289-306 (2006)

[17] Rätz, J.: On a functional equation related to projections of Abelian groups. Aequationes Math. 70(3), 279-297 (2005)

Pál Burai

Department of Applied Mathematics and Probability Theory University of Debrecen

Pf. 12

4010 Debrecen

Hungary

e-mail: burai@inf.unideb.hu

and

TU Berlin Institut für Mathematik

Str. des 17.Juni 136

10623 Berlin

Germany

e-mail: burai@math.tu-berlin.de

Attila Házy

Department of Applied Mathematics

University of Miskolc

3515 Miskolc-Egyetemváros

Hungary

e-mail: matha@uni-miskolc.hu

Tibor Juhász

Institute of Mathematics and Informatics

Eszterházy Károly College

Leányka út 4

3300 Eger

Hungary

e-mail: juhaszti@ektf.hu

Received: November 29, 2011

Revised: April 4, 2013 